\title{
Tympanic Membrane Collagen Expression by Dynamically Cultured Human Mesenchymal Stromal Cell/Star-Branched Poly( $\varepsilon$-Caprolactone) Nonwoven Constructs
}

\author{
Stefania Moscato ${ }^{1}$, Antonella Rocca ${ }^{1}$, Delfo D'Alessandro ${ }^{2}$, Dario Puppi ${ }^{3}{ }^{1}$, \\ Vera Gramigna ${ }^{4,5}$, Mario Milazzo ${ }^{5}$ D , Cesare Stefanini ${ }^{5}$, Federica Chiellini ${ }^{3}$, Mario Petrini ${ }^{1}$, \\ Stefano Berrettini ${ }^{2}$ and Serena Danti ${ }^{5,6, *(\mathbb{D})}$ \\ 1 Department of Clinical and Experimental Medicine, University of Pisa, via Savi 10, 56126 Pisa, Italy \\ 2 Department of Surgical, Medical, Molecular Pathology and Emergency Medicine, University of Pisa, \\ via Savi 10, 56126 Pisa, Italy \\ 3 Department of Chemistry and Industrial Chemistry, University of Pisa, via G. Moruzzi 13, 56124 Pisa, Italy \\ 4 Neuroscience Centre, University "Magna Graecia", viale Europa, 88100 Catanzaro, Italy \\ 5 The Biorobotics Institute, Scuola Superiore Sant'Anna, via R. Piaggio 34, 56025 Pontedera (PI), Italy \\ 6 Department of Civil and Industrial Engineering, University of Pisa, Largo L. Lazzarino 2, 56122 Pisa, Italy \\ * Correspondence: serena.danti@unipi.it; Tel.: +39-050-2217874
}

Received: 24 March 2020; Accepted: 23 April 2020; Published: 27 April 2020

check for updates

Featured Application: Our work shows the application of tissue engineering to regenerate in vitro the tympanic membrane by using human mesenchymal stromal cells from the bone marrow and a scaffold with suitable physico-mechanical properties and growth/differentiating factors. The possibility of regenerating a functional autologous eardrum substitute in laboratory can open new reconstructive routes in otologic surgery for patients with extensive tympanic membrane perforations due to traumas or chronic otitis.

\begin{abstract}
The tympanic membrane (TM) primes the sound transmission mechanism due to special fibrous layers mainly of collagens II, III, and IV as a product of TM fibroblasts, while type I is less represented. In this study, human mesenchymal stromal cells (hMSCs) were cultured on star-branched poly( $\varepsilon$-caprolactone) ( $\left.{ }^{*} \mathrm{PCL}\right)$-based nonwovens using a TM bioreactor and proper differentiating factors to induce the expression of the TM collagen types. The cell cultures were carried out for one week under static and dynamic conditions. Reverse transcriptase-polymerase chain reaction (RT-PCR) and immunohistochemistry (IHC) were used to assess collagen expression. A Finite Element Model was applied to calculate the stress distribution on the scaffolds under dynamic culture. Nanohydroxyapatite (HA) was used as a filler to change density and tensile strength of *PCL scaffolds. In dynamically cultured *PCL constructs, fibroblast surface marker was overexpressed, and collagen type II was revealed via IHC. Collagen types I, III and IV were also detected. Von Mises stress maps showed that during the bioreactor motion, the maximum stress in *PCL was double that in HA/*PCL scaffolds. By using a *PCL nonwoven scaffold, with suitable physico-mechanical properties, an oscillatory culture, and proper differentiative factors, hMSCs were committed into fibroblast lineage-producing TM-like collagens.
\end{abstract}

Keywords: eardrum; electrospinning; star-branched; poly( $\varepsilon$-caprolactone); bioreactor; fibroblast differentiation; collagen type II; mesenchymal stem cells; tympanoplasty; biomimetic 


\section{Introduction}

The tympanic membrane (TM), or eardrum, plays a fundamental role in sound transmission, its unique anatomical and physical features being ideal to function in varying frequency ranges. The human TM has been morphologically and functionally described since the 1970s [1,2]. Its development starts during the 28th week of gestation and arises from the most medial aspect of the meatal plug (basically, a cartilaginous tissue), which eventually becomes the external layer of the TM [3]. The fully developed TM is a three-layer structure, consisting of two histo-morphologically distinct areas-pars tensa, the largest, and pars flaccida, the thickest $[4,5]$. The outer and inner linings of the pars tensa are made up of epithelia, a keratinizing epithelium, and a simple cuboidal epithelium, respectively [4]. In between these two epithelia, a structural layer named lamina propria is located, which is connective tissue proper. In the pars tensa, the connective tissue is composed of collagen fibers with unique spatial arrangements, radial on the outer and circular on the inner side, in addition to parabolic fibers, which altogether build up a stiff and thin structure [5,6]. As a consequence, the peculiar fibrous network in the lamina propria of the pars tensa plays a key role in the physiology of the normal TM, and its damage after perforations, traumas, and infections generates a functional impairment [1]. Moreover, many studies have revealed that the vibration pattern of the TM reflects the underlying fiber bundle arrangement and thickness of the TM, thus confirming the tight connection between anatomy and physiology [7].

Immunohistochemical studies of the TM have shown that the fibrous layers in the lamina propria of the pars tensa are formed mainly by collagen types II and III, while collagen type I is present to a lesser extent $[1,8,9]$. Collagen type I has been found in the tympanic anulus (namely, the insertion zone to the meatus bone), whereas procollagens I and III in the subepidermal and submucosal connective tissue layers [1,8]. Collagen IV and other proteins are also found in TM [10].

The very high presence of collagen type II may be explained by referring to the embryological development of TM from the first branchial arch, which will give rise to fibrous stratum [3]. Each TM layer derives from a different embryonic leaflet (i.e., outer ectoderm, middle mesoderm and inner endoderm), which altogether interact to generate a functional eardrum through several transformations of the branchial arch mesenchyme, including chondrogenesis, that induces malleus elongation [11]. The complex collagenous network deriving from eardrum development has been considered to be the key for an efficient vibratory function of the TM [6-8]. In adults, apart from the typical banded fibrillar circular structure, which is characteristic of collagen organization, squared-section fibrils have also been observed in TM cross sections [1,5]. These structures, also found in other ear compartments, have revealed to be collagen type II [1,12]. In the eardrum, the overall collagenous structure is produced by TM fibroblasts, unique in the human body to synthetize collagen type II at a larger extent and collagen type I at a lesser extent [4].

Otitis media and perforation are among the most widespread pathologies of the TM [13]. If perforations are not able to self-repair, chronic infections can take place, which ultimately cause hearing loss and may require surgical treatment [14]. To date, autograft taken from connective tissue, such as the temporalis fascia and tragal cartilage are considered the gold standard materials for TM replacement [15]. In general, the outcome quality is related to the extent of the replacement, and for large perforations suboptimal outcomes have usually been reported [16].

In the last few years, a number of innovative strategies have been proposed for eardrum replacement and repair, including tissue engineering approaches, 3D-printed eardrums, growth factor injection, and stem cell therapy [17-22].

From a tissue engineering perspective, three pillars are needed: biomaterials in the form of porous scaffold (usually polymer-based), cells (usually, stem cells), and stimuli (e.g., chemical, mechanical and topographical). Among the many bioresorbable polymers available for tissue engineering scaffolding, star-branched polymers offer novel interesting options. They are made up by several linear polymeric chains centered in a small core unit; as such, their properties may greatly differ from those of their purely linear counterparts with the same molecular weight. The key advantages of star-polymers are their small size, compactness, and 3D structure, which ultimately affect viscosity and large possibility 
of functionalization through chain ends [23]. In particular, due to reduced hydrodynamic volume, leading to strong chain entanglement, star-branched poly( $\varepsilon$-caprolactone) $\left({ }^{*} \mathrm{PCL}\right)$ was found suitable for the electrospinning process $[24,25]$.

Electrospinning is a nanotechnology platform to produce nonwovens, with fiber size ranging from tens of nanometers up to a few microns, which complies with the dimensions of the protein fibers and fiber bundles present in the connective tissue, such as collagens [26]. Recent strategies of TM tissue engineering have focused on autologous approaches, stressing the use of undifferentiated cell sources, like mesenchymal stem (or stromal) cells (MSCs) [19,27]. MSCs isolated from the bone marrow have been processed in vitro towards many mature lineages of connective tissue, including fibroblasts, which hold importance for tendon and ligament regeneration [28]. It is widely known that human MSCs (hMSCs) can express collagen type II gene and protein under chondrogenic differentiation [29]. Dynamic culture systems based on bioreactors have been developed for mechanically responsive tissues (e.g., bone) and have shown interesting benefits [30]: they increase cell viability and scaffold spatial colonization due to the damping of mass transfer limitation in vitro, which represents a crucial issue for metabolically active tissues in the absence of blood supply. Additionally, dynamic cultures of tissue engineered constructs containing differentiating MSCs resulted in an enhanced cell maturation, as a direct consequence of the mechanical stimuli; finally, the spatial orientation of cells and extracellular matrix (ECM) components have been strongly related to applied stresses. In a previous study, we proposed electrospun poly(ethylene oxide terephthalate)/poly(butylene terephthalate) (PEOT/PBT) nonwovens, as scaffolds for eardrum regeneration, which were cultured with hMSCs in a TM bioreactor (WO/2015/040554) under non-differentiating conditions, demonstrating increased cell viability and penetration in the mesh thickness [27].

This study aims at the in vitro regeneration of a connective-like tissue mimicking the collagen types of the lamina propria of the pars tensa in a human TM. We used a tissue engineering approach, consisting of (i) a fibrous bioresorbable polymer scaffold; (ii) hMSCs; and (iii) biochemical, physical, and mechanical stimuli to differentiate hMSCs into fibroblasts synthetizing TM-like collagens. The mechanical stimuli are given via a TM bioreactor (WO/2015/040554) able to generate a hydrodynamic pressure and a tangential stress field in a membrane, thus inducing circular and radial strains during hMSC differentiation. Such a stress/deformation field aims to mimic the circular and radial patterns found in TM collagen fibers, thus inducing a physical stimulation over the cells at the scaffold interface. We hypothesized that, as a response to the mechanical stresses experienced by hMSCs within star-branched poly( $\varepsilon$-caprolactone) ( $\left.{ }^{*} \mathrm{PCL}\right)$ nonwoven scaffold (i.e., physical stimuli) and proper differentiating factors (i.e., biochemical stimuli), hMSCs would synthesize collagen types II, in addition to collagen type I and III. To investigate the specific physico-mechanical effect played by the electrospun scaffold toward hMSC differentiation in dynamic conditions, in silico tests, using Finite Element Modeling supported by experimental data, were performed by comparing a plain *PCL scaffold with a nanohydroxyapatite (HA)/*PCL composite counterpart, which showed a very similar architecture but diverse density and tensile modulus.

The possibility of comprehensively regenerating the TM collagen types represents a relevant step forward to the production of autologous and functional eardrum substitutes via a tissue engineering approach.

\section{Materials and Methods}

\subsection{Materials}

Three-arm star-branched poly( $\varepsilon$-caprolactone) $\left({ }^{*} \mathrm{PCL}\right)$, hydroxyapatite $(\mathrm{HA})$, Alpha-Modified Eagle's medium ( $\alpha$-MEM), Dulbecco's Modified Eagle Medium/F-12 (DMEM/F-12), trypsin, EDTA, $\mathrm{NaCl}$, Neutral red, Trypan blue, gelatin (type B, from bovine skin) Xylene, Triton X-100, mouse monoclonal anti-human fibroblast (F4771), bovine serum albumin (BSA), Mayer's hematoxylin solution, dibutylphthalate polystyrene xylene (DPX) mounting medium, methanol and acetone were 
purchased from Sigma-Aldrich (St. Louis, MO, USA); absolute ethanol was purchased from Carlo Erba (Milan, Italy); Dulbecco's phosphate buffered saline (D-PBS), penicillin, streptomycin, fluconazole were purchased from Lonza (Milan, Italy); Transforming Growth Factor beta 1 (TGF- $\beta 1$ ), ascorbic acid, heat-inactivated fetal bovine serum (FBS), L-Glutamine, Rhodaminate Sytox green, Rhodaminate phalloidin were purchased from Invitrogen (Carlsbad, CA, USA); RNeasy ${ }^{\circledR}$ Plus Mini kit was purchased from Qiagen (Hilden, Germany); SsoFast ${ }^{\mathrm{TM}}$ EvaGreen $^{\circledR}$ supermix, iScript ${ }^{\mathrm{TM}} \mathrm{cDNA}$ synthesis kit were purchase from Bio-Rad Laboratories (Hercules, CA, USA); neutral buffered formalin, optimal cutting temperature (OCT) medium were purchased from Bio-Optica (Milan, Italy); paraffin Histoplast LP was purchased from Thermo Fisher Scientific (Waltham, MA, USA); Citrate buffer was purchased from Diapath (BG, Italy); mouse monoclonal anti-human collagen type II (sc52658) was purchased from Santa Cruz Biotechnologies (Santa Cruz, CA, USA); rabbit polyclonal anti-human collagen type I (ab34710), mouse monoclonal anti collagen type III (ab6310), mouse monoclonal anti collagen type IV (ab6311) were purchased from AbCam (Cambridge, UK). Normal goat serum, goat anti-mouse secondary antibody, Vectastain Elite ABC Kit Standard were purchased from Vector Lab (Burlingame, CA, USA); chromogen substrate 3-3' diaminobenzidine tetrahydrochloride (DAB) was purchased from Amresco (Solon, OH, USA).

\subsection{Methods}

\subsubsection{Scaffold Preparation}

${ }^{*} \mathrm{PCL}$ in powder was dissolved in acetone at a concentration of $15 \% w / v$ at $33^{\circ} \mathrm{C}$ under stirring until a homogeneous solution was achieved. To obtain HA-loaded ${ }^{*} \mathrm{PCL}$, the polymeric solution prepared as above was cooled for $10 \mathrm{~min}$ RT and added with $\mathrm{HA}$ in a ratio of 1:4 with respect to the weight in grams of ${ }^{*} \mathrm{PCL}$. The suspension obtained was then left under stirring at $35^{\circ} \mathrm{C}$ for $30 \mathrm{~min}$. The polymeric solutions of ${ }^{*} \mathrm{PCL}$ and $\mathrm{HA} /{ }^{*} \mathrm{PCL}$ were placed in gas tight syringes with a needle having an internal diameter of $0.84 \mathrm{~mm}$. To obtain collections of concentrated fibers, a configuration consisting of two parallel metal screens (distance between the two screens was $18 \mathrm{~cm}$ ) was used, one functioning as a fiber collector and the other as an auxiliary electrode [31]. The fluid was supplied with a syringe through a metal needle projecting from a screen and the fibers were collected on the other grounded screen. A voltage of $25 \mathrm{kV}$ was applied on one screen and $0 \mathrm{kV}$ on the other so that a potential difference of $25 \mathrm{kV}$ was generated. The solutions were deposited with a flow rate of $8 \mathrm{~mL} / \mathrm{h}$ at RT on the grounded screen. After electrospinning, the fibers were placed in petroleum ether for $24 \mathrm{~h}$ to allow the elimination of residual solvents.

\subsubsection{Scaffold Characterization}

${ }^{*} \mathrm{PCL}$ and $\mathrm{HA} /{ }^{*} \mathrm{PCL}$ nonwovens were characterized as follows: morphology and composition via scanning electron microscopy (SEM) equipped with energy dispersive X-ray spectroscopy (EDX), density via pycnometry and mechanical properties via INSTRON dynamometry. For morphological analysis, the samples were mounted on aluminum stumps and sputter-coated with gold (Sputter Coater Emitech K550, Quorum Technologies Ltd., West Sussex, UK) and then were observed using a JSM-520 (Jeol Ltd., Tokyo, Japan) at 15-20 KV and 1000× magnification. EDX was used to detect HA particles in HA/*PCL nonwovens by investigating the presence of $\mathrm{Ca}$ and $\mathrm{P}$ elements. The scaffold density was calculated as the ratio between its mass, weighted with a digital scale and its volume, measured with a glass pycnometer using absolute ethanol as a non-solvent able to soak the sample, following best practice procedures. The scaffold mechanical properties were characterized following ASTM D 882-91, as from previous studies [24]. Briefly, a dog bone-shaped template was used to cut samples out from visible defect-free parts of the meshes. Their average thickness was calculated from five measurements taken by means of a micrometer (Vogel S.r.l., Leno (BS), Italy) in the load-bearing part of each sample. Tensile characterization was performed until break, at room temperature and 
ambient humidity, by testing five replicates at a constant extension rate of $2 \mathrm{~mm} / \mathrm{min}$. The elastic modulus was calculated as the slope of the resulting stress-strain curve in the linear region.

\subsubsection{Scaffold Sterilization}

Fiber scaffolds, previously cut into discs to fit 24-well plates or the bioreactor holder (surface = $2.2 \mathrm{~cm}^{2}$ ), were sterilized by incubation in absolute ethanol for $12 \mathrm{~h}$. Subsequently the scaffolds were washed three times in sterile D-PBS added with $300 \mathrm{U} / \mathrm{mL}$ penicillin/streptomycin and $3 \%$ fluconazole and finally soaked in sterile D-PBS without antibiotics. Before cell seeding, scaffolds were aspirated from the washing buffer and let dry under the biohood.

\subsubsection{Ethical Statement}

HMSCs were obtained from bone marrow of the femoral medullary canal of patients admitted to Pisa hospital for orthopedic surgery. Briefly, during the surgical preparation of the medullary canal to house the prosthetic stem for a total hip replacement surgery, the marrow blood overflowing (about $10-15 \mathrm{~mL}$, depending on the prosthesis size) was recovered instead of being discharged. Samples were collected after informed consent, treated anonymously and in conformity to the principles expressed by the Declaration of Helsinki. Human TMs were explanted from a formalin-fixed cadaveric temporal bone purchased from International Biologicals Inc. (Detroit, MI, USA) for research use only.

\subsubsection{Cell Isolation and Seeding on the Scaffolds}

HMSCs were isolated from bone marrow aspirates as previously described [19,27,32]. After 10-15 days from the seeding, hMSCs were detached from flasks by a D-PBS solution containing $0.5 \%$ $v / v$ trypsin, $0.2 \% v / v$ EDTA, $0.09 \% w / v \mathrm{NaCl}$ for $5 \mathrm{~min}$ at $37^{\circ} \mathrm{C}$, and then were centrifuged $10 \mathrm{~min}$ at $1000 \mathrm{rpm}$ and expanded by plating them at the density of $1000 \mathrm{cell} / \mathrm{cm}^{2}$ using a growth culture medium (CM) consisting of $\alpha$-MEM supplemented with $10 \%$ (v\%) FBS, $2 \mathrm{mM} \mathrm{L-glutamine,} 100 \mathrm{IU} / \mathrm{mL}$ penicillin, $100 \mathrm{mg} / \mathrm{mL}$ streptomycin, and $1 \%$ fluconazole. Once the semi-confluence was reached, hMSCs were trypsinized and viable cells were counted with a hemocytometer, using Trypan Blue exclusion dye. HMSCs, suspended in $2 \%(w / v)$ gelatin/water solution, were seeded on the scaffolds at a density of 240,000 viable cells per $\mathrm{cm}^{2}$ of scaffold area. HMSC/scaffold constructs were placed in 24-well or six-well plates and cultured in incubator for seven days at $37^{\circ} \mathrm{C}$ and in humidified $95 \% / 5 \%$ air $/ \mathrm{CO}_{2}$ environment. HMSCs were also seeded at density of 10,000 cell/well on chamber slides and cultured for one week. Growth (non-differentiating) CM was replaced every three days for one week, before switching to differentiating CM.

\subsubsection{Construct Differentiation}

To differentiate hMSCs toward the TM fibroblastic phenotype a CM consisting of DMEM/F-12 added with $10 \%$ FBS, $10 \mathrm{ng} / \mathrm{mL}$ of TGF- $\beta 1,50 \mathrm{ng} / \mathrm{mL}$ of ascorbic acid, $200 \mathrm{mM}$ L-Glutamine, $100 \mathrm{U} / \mathrm{mL}$ penicillin, $0.1 \mathrm{mg} / \mathrm{mL}$ streptomycin and $1 \%$ fluconazole was used. After one week of culture in non-differentiating conditions, the constructs were placed inside a bioreactor, and the culture was continued for four additional weeks. The dynamic culture system (bioreactor) used in this study was a patented apparatus allowing the vertical oscillatory motion of the cell/scaffold samples in a CM filled tube at $0.23 \mathrm{~Hz}$ (WO/2015/040554). Subsets of samples were cultured in non-differentiating CM, as negative controls. At the endpoint, the samples were processed for cell viability and morphological analyses, i.e., SEM, confocal laser scanning microscopy (CLSM), and immunohistochemistry. Construct cultures, both static and dynamic, were carried out in standard conditions, namely $37^{\circ} \mathrm{C}$ and in $95 \% / 5 \%$ humidified air $/ \mathrm{CO}_{2}$ incubator. 


\subsubsection{Neutral Red Assay}

Neutral Red viability assay is based on the accumulation of this dye in the lysosomes of living cells that have not altered plasma membranes. To perform the assay, the dye was dissolved in CM at a $50 \mu \mathrm{g} / \mathrm{mL}$ concentration at $37^{\circ} \mathrm{C}$ overnight. The cell/scaffold samples were added with the sterile-filtered solution of Neutral Red/CM and placed in the incubator for $1 \mathrm{~h}$. After incubation, the samples were washed in D-PBS, and observed under a stereomicroscope equipped with a digital camera.

\subsubsection{Gene Expression Analysis}

RNA extraction was performed using the RNeasy ${ }^{\circledR}$ Plus Mini commercial kit, following the manufacturer's instructions. cDNA synthesis reaction was performed using the iScript ${ }^{\mathrm{TM}} \mathrm{cDNA}$ synthesis kit in according to the manufacturer's instructions. The reverse transcriptase used is the Moloney murine leukemia virus reverse transcriptase (MMLV-RT). The reverse transcription reaction was performed using a $20 \mu \mathrm{L}$ final volume solution containing $4 \mu \mathrm{L} 5 \mathrm{x}$ iScript reaction mix, $1 \mu \mathrm{L}$ iScript reverse transcriptase, nuclease-free water $/ \mu \mathrm{L}, 250 \mathrm{ng} \mathrm{RNA} / \mu \mathrm{L}$. The synthesis program consisted in an incubation at $25^{\circ} \mathrm{C}$ for $5 \mathrm{~min}$, followed by an incubation at $42{ }^{\circ} \mathrm{C}$ for $30 \mathrm{~min}$ and at $85^{\circ} \mathrm{C}$ for $5 \mathrm{~min}$. EvaGreen kit was used to perform the Real Time PCR analysis. The amplification reactions were performed on an iCycler-iQ5 Optical System thermocycler (Bio-Rad Laboratories, Hercules, CA, USA). Each amplification reaction was set up in $20 \mu \mathrm{L}$ containing $10 \mu \mathrm{L}$ of SsoFast ${ }^{\mathrm{TM}}$ EvaGreen $^{\circledR}$ supermix, $4 \mu \mathrm{L}$ primer (primer forward (Fw) and reverse (Rv) $2 \mu \mathrm{M}$ ), $2 \mu \mathrm{L}$ cDNA diluted 1: 4, $\mathrm{H}_{2} \mathrm{O}$. The temperature program used was one cycle at $95^{\circ} \mathrm{C}$ for $30 \mathrm{~s}$ to activate the enzyme, 40 cycles at $95^{\circ} \mathrm{C}$ for $5 \mathrm{~s}$ and at $60^{\circ} \mathrm{C}$ for $10 \mathrm{~s}$. The specificity of the amplification reaction and the presence of dimers were evaluated by melting curve analysis. The samples were analyzed in triplicate $(n=3)$ and the housekeeping gene RPLP0 was also amplified, in addition to the target gene, to evaluate possible variations in the amount of cDNA used and thus to normalize the values. The target genes evaluated are collagen type I- $\alpha 1$, collagen type II- $\alpha 1$, collagen type IV- $\alpha 1$; and collagen type IV- $\alpha 2$. The primer sequences are reported in Table 1.

Table 1. Analyzed genes involved in differentiation of mesenchymal stem cells (MSCs) toward tympanic membrane (TM) fibroblast phenotype seeded on star-branched poly ( $\varepsilon$-caprolactone) ( ${ }^{*} \mathrm{PCL}$ ) scaffolds; primer sequence for RPLP0 housekeeping gene, product base pair and applied temperature (Tm).

\begin{tabular}{cccrc}
\hline Gene & Acronym & Primer Sequence $\left(\mathbf{5}^{\prime}-\mathbf{3}^{\prime}\right)$ & Product $(\mathbf{b p})$ & Tm $\left({ }^{\circ} \mathbf{C}\right)$ \\
\hline Collagen type I- $\alpha 1$ & COL1A1 & AGACATCCCACCAATCAC & 118 & 60.5 \\
& & GTCATCGCACAACACCTT & 62.1 & 63.9 \\
Collagen type II- $\alpha 1$ & COL2A1 & GAGCAGCAAGAGCAAGGA & 135 & 64.0 \\
& & GACAGCAGGCGTAGGAAG & 168 & 61.5 \\
Collagen type IV- $\alpha 1$ & COL4A1 & CGCAAGTTCAGCACAATG & 125 & 61.1 \\
Collagen type IV- $\alpha 2$ & COL4A2 & ACTGGTGATTTCGGTGAC & 60.6 \\
Ribosomal protein large, P0 & RPLP0 & CCTTCTGTTCCCTTCTCTC & 95 & 63.6 \\
& & GCAGCAGCTGGCACCTTATTG & 63.3 \\
\hline
\end{tabular}

The levels of gene expression were evaluated by the Pfaffl method, which combines gene quantification and normalization in a single calculation [33]. The model incorporates the amplification efficiencies of the target and control genes to correct the differences between the two analyses. The relative expression ratio $(R)$ of the target gene is calculated based on both efficiency $(E)$ and threshold cycle $(\mathrm{Ct})$ difference of an unknown and a control sample. This ratio was thus expressed by comparison to the reference gene.

\subsubsection{Sample Preparation for Morphological and Histological Analysis}

After one week of culture in differentiative medium, the constructs were fixed in $4 \% w / v$ neutral buffered formalin at $4{ }^{\circ} \mathrm{C}$ overnight. The next day, after washings in D-PBS, the specimens were 
embedded in OCT cryogenic medium at $-80{ }^{\circ} \mathrm{C}$ for one day and then sectioned into 8 - $\mu \mathrm{m}$ slices by a standard cryostat. HMSC cultured in chamber slide were fixed in $1 \% w / v$ neutral buffered formalin at $4{ }^{\circ} \mathrm{C}$ for $10 \mathrm{~min}$ and air dried. Human TMs, explanted from a fixed cadaveric temporal bone sample were paraffin embedded, dehydrated using $70 \%(\mathrm{v} \%), 80 \%(\mathrm{v} \%)$, and $95 \%(\mathrm{v} \%)$ ethanol/water solutions (30 min, $30 \mathrm{~min}$, and twice $45 \mathrm{~min}$ ) and absolute ethanol (three times for $1 \mathrm{~h}$ ) in a thermostatic bath set at $40{ }^{\circ} \mathrm{C}$. Therefore, TM samples were clarified in xylene twice for $45 \mathrm{~min}$ at $40{ }^{\circ} \mathrm{C}$ and rinsed in liquid paraffin at $60^{\circ} \mathrm{C}$ for $2 \mathrm{~h}$. Finally, the specimens were paraffin-embedded and sectioned into 8 - $\mu \mathrm{m}$ slices by standard microtome.

\subsubsection{CLSM Analysis}

After formalin fixation, hMSC/scaffold samples were permeabilized in $0.2 \% v / v$ TritonX-100 for $5 \mathrm{~min}$, incubated in Sytox green for $10 \mathrm{~min}$ and phalloidin linked to rhodamine for $45 \mathrm{~min}$ following the manufacturer's instructions. After staining, the samples were observed under a confocal microscope Nikon Eclipse TU2000-U (Nikon Instruments, Amsterdam, The Netherlands) and stacks were acquired at intervals of a few microns along their thickness ( $z$ axis). The scans collected were processed with the Nikon ACTU software to adjust the color levels of the channels and were superimposed using the Image-J software (version 1.46 r; http://imagej.nih.gov, National Institute of Health-NIH, Bethesda, MD, USA).

\subsubsection{Immunohistochemistry}

Construct sections as well as hMSCs cultured in chamber slide were washed twice in double-distilled (dd) water and rinsed in D-PBS for 5 min; TM sections were deparaffinized by xylene (twice for $7 \mathrm{~min}$ ), rehydrated in absolute ethanol (three times for $7 \mathrm{~min}$ ), washed in dd- $\mathrm{H}_{2} \mathrm{O}$ and finally rinsed in D-PBS for $5 \mathrm{~min}$. All the samples were treated to detect the expression of the following proteins: fibroblast surface marker, collagens type I, type II, type III, and type IV.

To reveal collagen type II in hMSC/scaffold constructs and TM sections, incubation in citrate buffer in a thermostatic bath at $90^{\circ} \mathrm{C}$ for $10 \mathrm{~min}$ was necessary. The specimens were permeabilized by using $0.2 \% v / v$ Triton $\mathrm{X}-100$ for $10 \mathrm{~min}$. To quench the endogen peroxidases, after D-PBS washing, the samples were incubated in a methanol solution containing $0.6 \% v / v \mathrm{H}_{2} \mathrm{O}_{2}$ at 36 volumes for $20 \mathrm{~min}$ in the dark. After washings, in order to block unspecific binding sites of secondary antibody, the specimens were incubated in $5 \%$ normal goat serum for $20 \mathrm{~min}$ at $37^{\circ} \mathrm{C}$. Finally, the sections and hMSCs in chamber slide were incubated with primary antibodies directed against specific antigens and diluted in a D-PBS solution containing $0.1 \% w / v$ BSA. Incubation was performed in humidified chamber overnight at $4{ }^{\circ} \mathrm{C}$.

The primary antibodies employed were: rabbit polyclonal anti-human collagen type I diluted 1:16,000, mouse monoclonal anti collagen type III diluted 1:300, mouse monoclonal anti-human collagen type II diluted 1:50, mouse monoclonal anti collagen type IV diluted 1:70; mouse monoclonal anti-human fibroblast surface marker diluted 1:100 in hMSC culture, 1:800 in TM sections and 1:6000 in construct sections; negative controls were obtained by incubating some sections with $0.1 \%$ BSA/D-PBS solution only.

The subsequent day, specimens were incubated with biotynilated goat anti-mouse secondary antibody for $1 \mathrm{~h}$, then with peroxidase-streptavidin for $30 \mathrm{~min}$. Immunoreaction was revealed in brown after incubating the samples with chromogen substrate $\mathrm{DAB}$ activated with $\mathrm{H}_{2} \mathrm{O}_{2}$ for 5 min in the dark. Counterstaining was performed with Mayer's hematoxylin solution for $30 \mathrm{~s}$ followed by washing in tap water for $1 \mathrm{~min}$ in order to reveal in blue-violet cell nuclei counterstaining. Finally, the specimens were dehydrated in absolute ethanol (three times for $5 \mathrm{~min}$ ), clarified in xylene (three times for $5 \mathrm{~min}$ ) and mounted with coverslip using DPX mounting agent. In the second part of the reaction, washing in $0.01 \% v / v$ TritonX-100 were performed after each step. Immunohistochemical reaction was observed under a light microscope (DMD 108, Leica, Wetzlar, Germany) equipped with a digital camera. 
A semiquantitative analysis of antigenic positivity was performed by three independent observers according to the following criteria: $-=$ negative; $+/-=$ weak positivity; $+=$ good positivity $+++=$ strong positivity; $++++=$ very strong positivity.

\subsubsection{Computational Analysis of Bioreactor Fluid Dynamics}

A finite element analysis within the perfusion bioreactor, carried out with Comsol Multiphysics v. 4.1 (COMSOL Multiphysics, Burlington, MA, USA) allowed for the evaluation of fluid dynamic parameters on the surface of the scaffold in contact with the fluid during the new-tissue growth. The objective is to verify the laminar flow regimen and to determine the shear stress acting on the cell/scaffold construct in distinct scaffold areas.

Fluid motion was modeled by using three-dimensional incompressible Navier-Stokes equations for Newtonian flows. The analysis consisted of two distinct procedures: (1) a fluid-dynamics analysis including a calculation of the velocity field in saline solution; (2) a mechanical analysis of the scaffold deformations. The two procedures were coupled. The main characteristics of the analysis are: (a) fluid dynamics analysis, in which the computational model solves the Navier-Stokes equations in a saline solution domain; (b) mechanical analysis, which takes into account only the domains related to the holder and the scaffold surface. Linear elastic and isotopic behavior was used for both solid materials. The scaffold and the material properties considered are shown in Tables S1 and S2. For the fluid-dynamic analysis, a constant velocity value - evaluated by tracking holder and scaffold movement, was adopted as an inlet boundary condition, while a pressure condition was used on the end of the test tube. As for the solid mechanics analysis, a prescribed displacement, evaluated by really tracking rings and scaffold movement, was used as boundary condition. An adaptive mesh is used because model geometry (rings and scaffold) is moving with time.

\subsubsection{Statistical Analysis}

Statistical analysis of gene expression was carried out using student $\mathrm{t}$ - test followed by Bonferroni as a "post-hoc" test. The analysis was conducted by comparing the data of independent experiments and the differences were considered statistically significant for $p<0.05$. Data were expressed as mean \pm standard deviation.

\section{Results}

\section{1. *PCL Scaffold Characterization}

The fabrication method allowed beadless anisotropic *PCL nonwovens to be obtained [24], made up by submicrometric fibers. HA/*PCL electrospun meshes showed similar aspect, with small beaded areas due to the presence of HA, as confirmed by EDX analysis (Figure S1). As a result of the diverse composition, plain *PCL and HA/*PCL resulted in different fabric density $\left(1.066 \mathrm{~g} / \mathrm{dm}^{3}\right.$ and $0.68 \mathrm{~g} / \mathrm{dm}^{3}$, respectively) and different Young's moduli (3.00 MPa and 1.39 MPa, respectively), as experimentally measured (Table S1).

\subsection{Viability of $h M S C{ }^{*} P C L$ Constructs}

Neutral red assay revealed the presence and spatial distribution of viable hMSCs in the ${ }^{*} \mathrm{PCL}$ scaffolds in all the culture conditions tested (Figure 1).

The constructs differentiated under dynamic conditions were removed from the bioreactor holder and cut into wedges to allow for multiple assays. HMSCs were localized in the central part of the scaffold (Figure 1c,d). The size of the scaffold to be used in the bioreactor was, indeed, cut larger than that of the scaffolds to be used in static conditions to provide enough lateral space for the bioreactor holder. In the dynamically cultured constructs, the neutral red intensity appeared generally higher than in statically cultured constructs and showed circular-like hints with strong dye accumulation 
(Figure 1c,d). Non-differentiated hMSCs in *PCL scaffolds appeared less uniformly distributed than their differentiated counterparts.
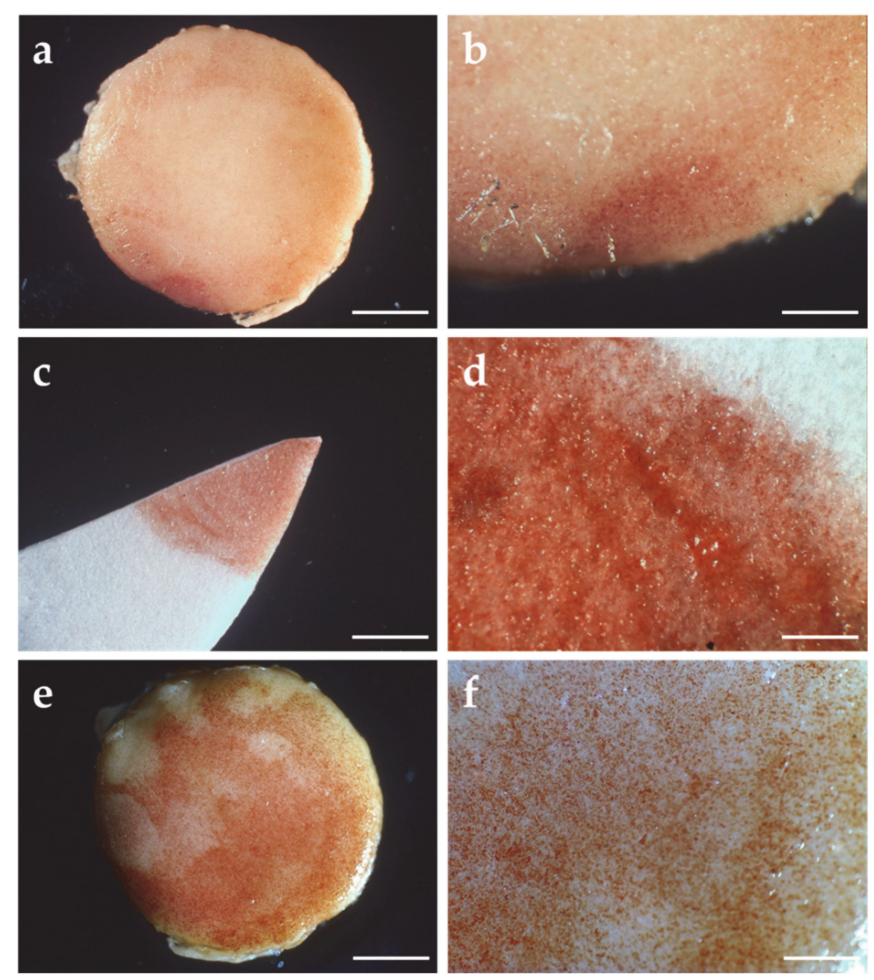

Figure 1. Neutral red assay show red-stained viable cells imaged via stereomicroscopy on human mesenchymal stromal cell (hMSC)/*PCL constructs: $(\mathbf{a}, \mathbf{b})$ differentiated in static conditions; $(\mathbf{c}, \mathbf{d})$ differentiated in dynamic conditions; and, (e,f) undifferentiated in static conditions. (a,c,e) Scale bar $=2 \mathrm{~mm} ;(\mathbf{b}, \mathbf{d}, \mathbf{f})$ scale bar $=600 \mu \mathrm{m}$.

\subsection{Gene Expression Evaluation in the hMSC/*PCL Constructs}

The expression levels of collagens type I, II, IV- $\alpha 1$ and IV- $\alpha 1$ in both differentiated and undifferentiated hMSCs on the ${ }^{*}$ PCL scaffolds under static or dynamic culture conditions were analyzed and compared after seven days (Figure 2).

Collagen type I mRNA expression was significantly increased in the constructs differentiated under static conditions if compared to the undifferentiated counterpart $(p<0.01)$. In the constructs differentiated under dynamic conditions, no statistically significant difference of Collagen type I mRNA expression was revealed if compared to the undifferentiated control. However, it was observed a statistically significant decrease if compared with the constructs differentiated under static conditions $(p<0.01)$ (Figure 2a). In both differentiated groups (statically and dynamically cultured), collagen type II mRNA expression was significantly higher than in the undifferentiated control $(p<0.05)$; however, no statistically significant differences could be detected between the differentiated groups (Figure $2 b$ ). Collagen type IV- $\alpha 1$ gene expression was increased in the constructs differentiated under static conditions if compared to the undifferentiated control $(p<0.05)$. Differently, in the constructs differentiated under dynamic conditions, no difference of mRNA expression was detected for this collagen type if compared to the undifferentiated control. A statistically significant decrease of mRNA expression was highlighted in dynamically differentiated samples if compared to those differentiated under static conditions $(p<0.05)$ (Figure $2 c$ ). Collagen type IV- $\alpha 2$ gene expression resulted to be modulated by neither the differentiating $\mathrm{CM}$ nor culture methods in our experimental conditions (Figure 2d). 

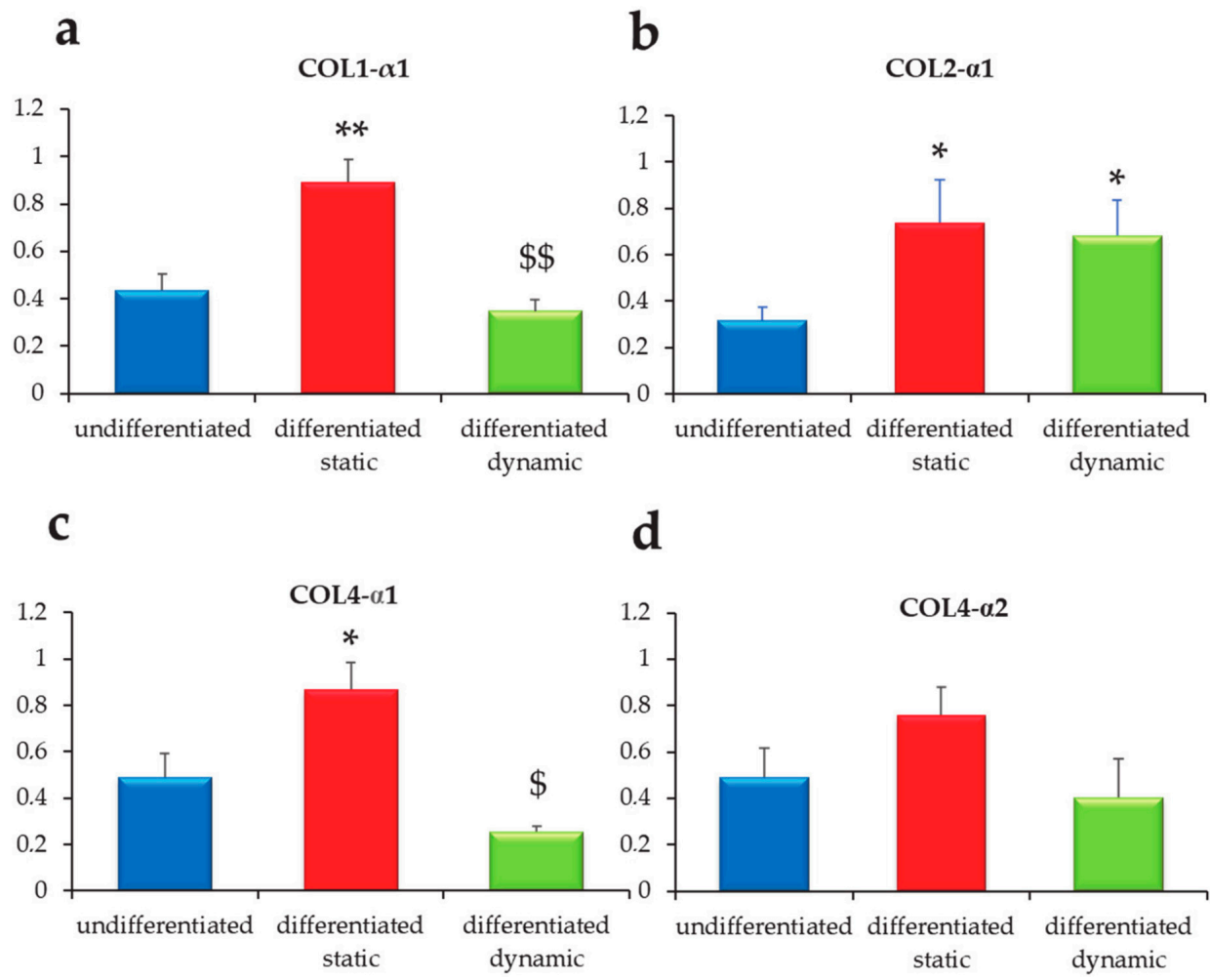

d

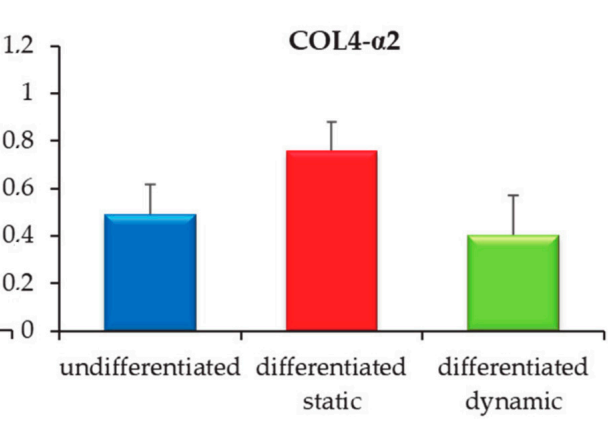

Figure 2. Collagen gene expression analysis performed on $\mathrm{hMSC} /{ }^{*} \mathrm{PCL}$ constructs for the following genes: (a) Col1- $\alpha 1$; (b) Col2- $\alpha$; (c) Col4- $\alpha 1$; and (d) Col4- $\alpha 2$ : (a-d) Statistical significance: ${ }^{*}=p<0.05$; ** $=p<0.01$ vs undifferentiated; $\$=p<0.05 ; \$ \$=p<0.01$ vs differentiated statically cultured hMSC/*PCL constructs.

\subsection{Morphological Analysis of the *PCL/MSC Constructs}

The morphological analysis of the cell/scaffold constructs performed by CLSM is shown in Figure 3.
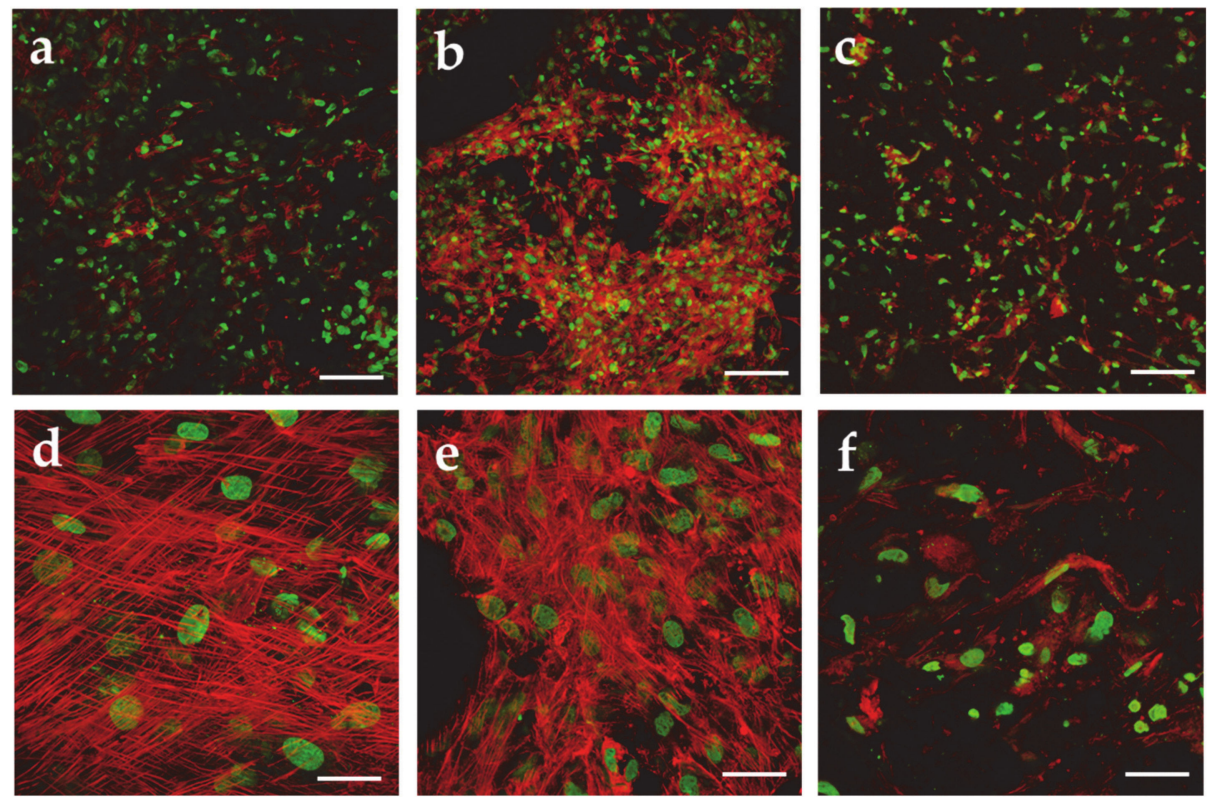

Figure 3. Confocal laser scanning microscopy (CLSM) analysis on hMSC/*PCL constructs how cell nuclei imaged in green and $f$-actin in red: $(\mathbf{a}, \mathbf{d})$ differentiated in static conditions; $(\mathbf{b}, \mathbf{e})$ differentiated in dynamic conditions; (c,f) undifferentiated. (a-c) Scale bar $=100 \mu \mathrm{m}$, original Magnification 200x; $(\mathbf{d}-\mathbf{f})$ Scale bar $=30 \mu \mathrm{m}$, original magnification $600 \times$. 
HMSCs were quite uniformly distributed over the entire surface of the scaffolds, cell nuclei were well visible and $f$-actin bundles in the cytoplasm were well extended. In particular, $f$-actin was more expressed in both differentiated construct types (Figure $3 a, b$ ), and less expressed in the undifferentiated controls (Figure $3 \mathrm{c}, \mathrm{f})$. Dynamically differentiated constructs showed the most intense $f$-actin expression (Figure $3 \mathrm{~b}, \mathrm{e})$, whereas statically differentiated ones showed a regular distribution of cytoskeletal filaments (Figure 3a,d).

\subsection{Immunohistochemical Analysis}

The outcomes of immunohistochemistry performed on hMSC/*PCL constructs in different culture conditions (i.e., statically differentiated, dynamically differentiated and undifferentiated), as well as undifferentiated hMSCs cultured in chamber slides and human TM samples used as baseline controls, are reported in Figures 4-6.

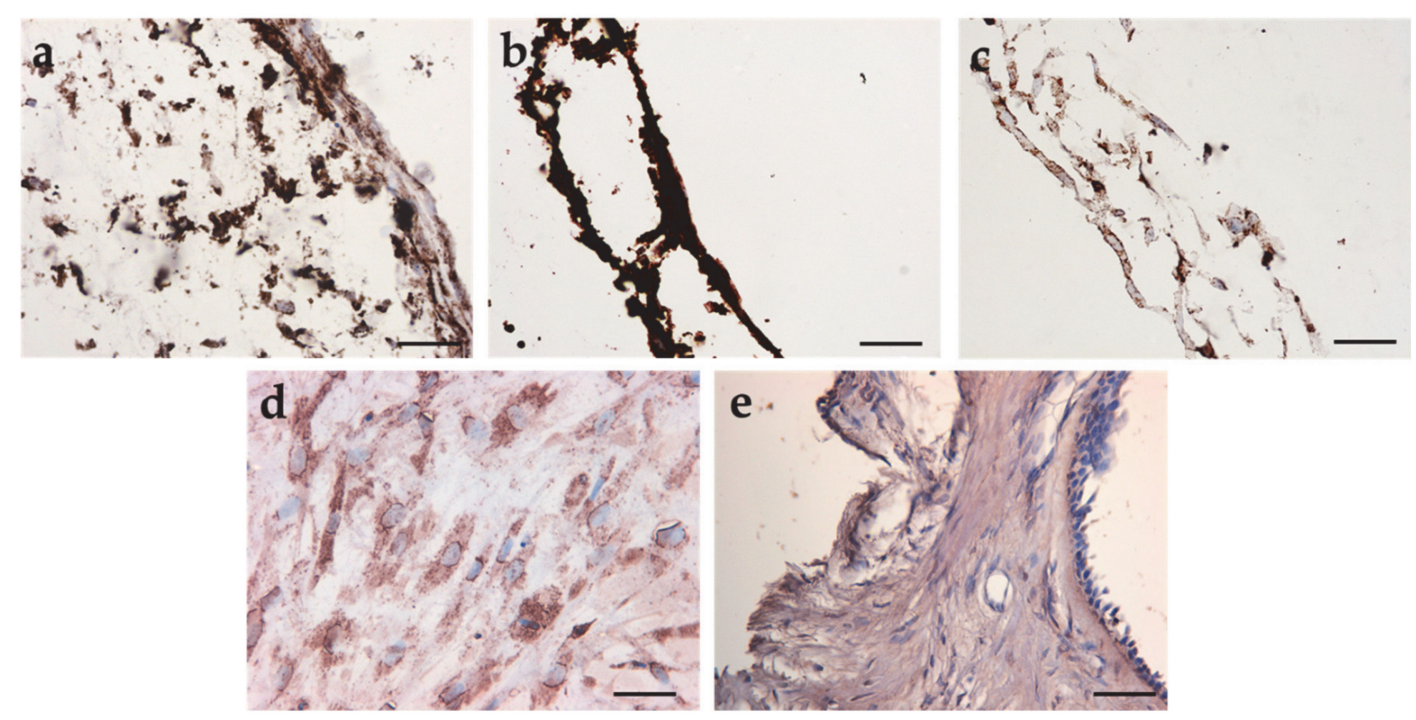

Figure 4. Immunohistochemical analysis of $\mathrm{hMSC} /{ }^{*} \mathrm{PCL}$ constructs showing fibroblast surface marker expression in brown and cell nuclei in violet as obtained via immunohistochemistry on: (a-c) hMSC/*PCL constructs [(a) statically differentiated, (b) dynamically differentiated, (c) undifferentiated]; (d) undifferentiated hMSCs cultured on chamber slides; (e) human TMs. (a-e) Scale bar is $50 \mu \mathrm{m}$. Original magnification $400 \times$.

Fibroblast surface marker resulted expressed in all the constructs, being in the differentiated ones more intensely expressed than in the undifferentiated counterpart (Figure 4). Moreover, in the constructs cultured under dynamic conditions, the fibroblast surface marker expression level was the highest one (Figure 4a-c). The expression level of this antigen in undifferentiated hMSCs cultured in chamber slides was present and comparable to the immunopositivity of the undifferentiated constructs (Figure $4 \mathrm{~d}$ ). The immunopositivity in native TMs was localized only in some areas of the ECM (Figure 4e) and was comparable to that of the undifferentiated statically cultured construct (Figure 4c).

Collagen type II expression is reported in Figure 5. Its expression was weak in all the constructs, but in the constructs cultured under dynamic conditions (Figure $5 \mathrm{a}-\mathrm{c}$ ). Undifferentiated hMSC cultured in chamber slide showed a good basal expression (Figure 5d) and native TMs showed very intense immunopositivity for this marker (Figure 5e). From these results, it is evident that only the combination of the differentiating cocktail and the bioreactor was able to increase the collagen type II expression in hMSCs (Figure 5b). 

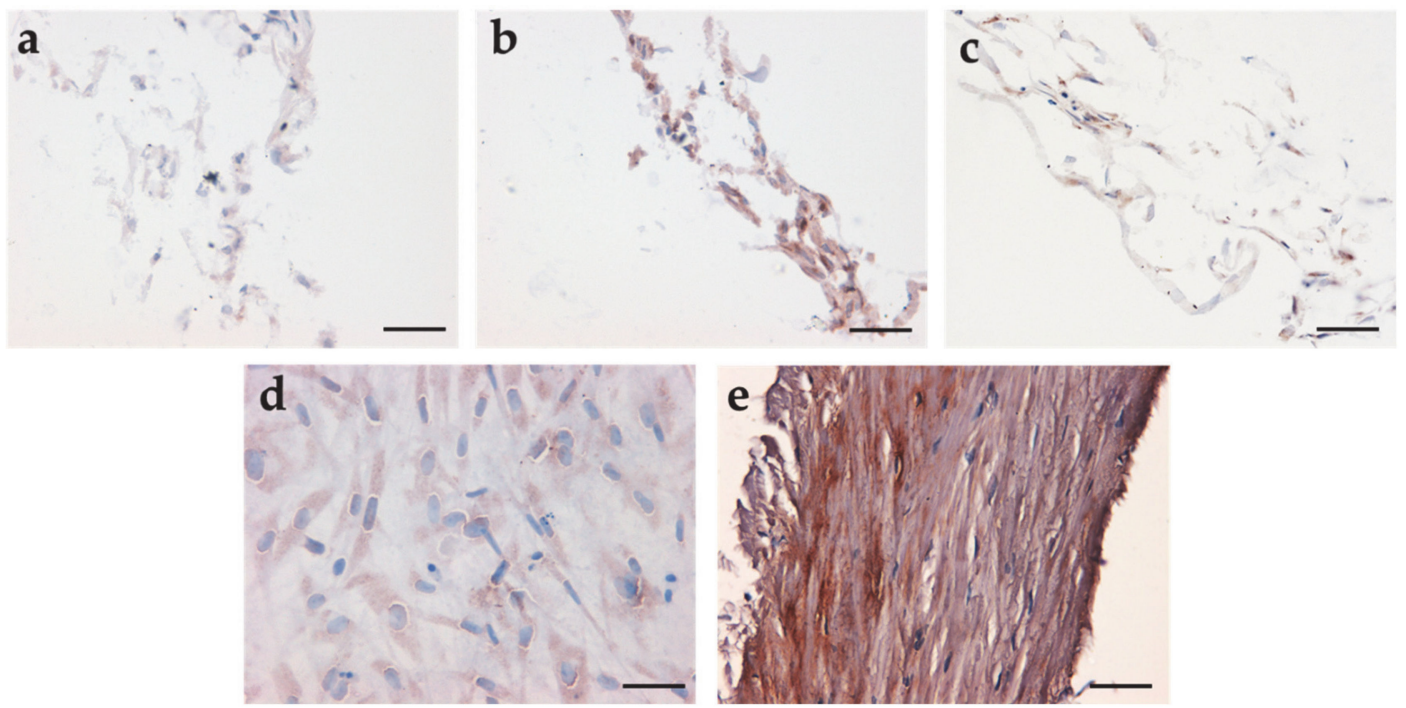

Figure 5. Immunohistochemical analysis of hMSC/*PCL constructs showing collagen type II expression in brown and cell nuclei in violet as obtained via immunohistochemistry on: $(\mathbf{a}-\mathbf{c})$ hMSC/ ${ }^{*}$ PCL constructs: (a) statically differentiated, (b) dynamically differentiated, (c) undifferentiated; (d) undifferentiated hMSCs cultured on chamber slides; (e) human TMs. (a-e) Scale bar is $50 \mu \mathrm{m}$. Original magnification 400x.

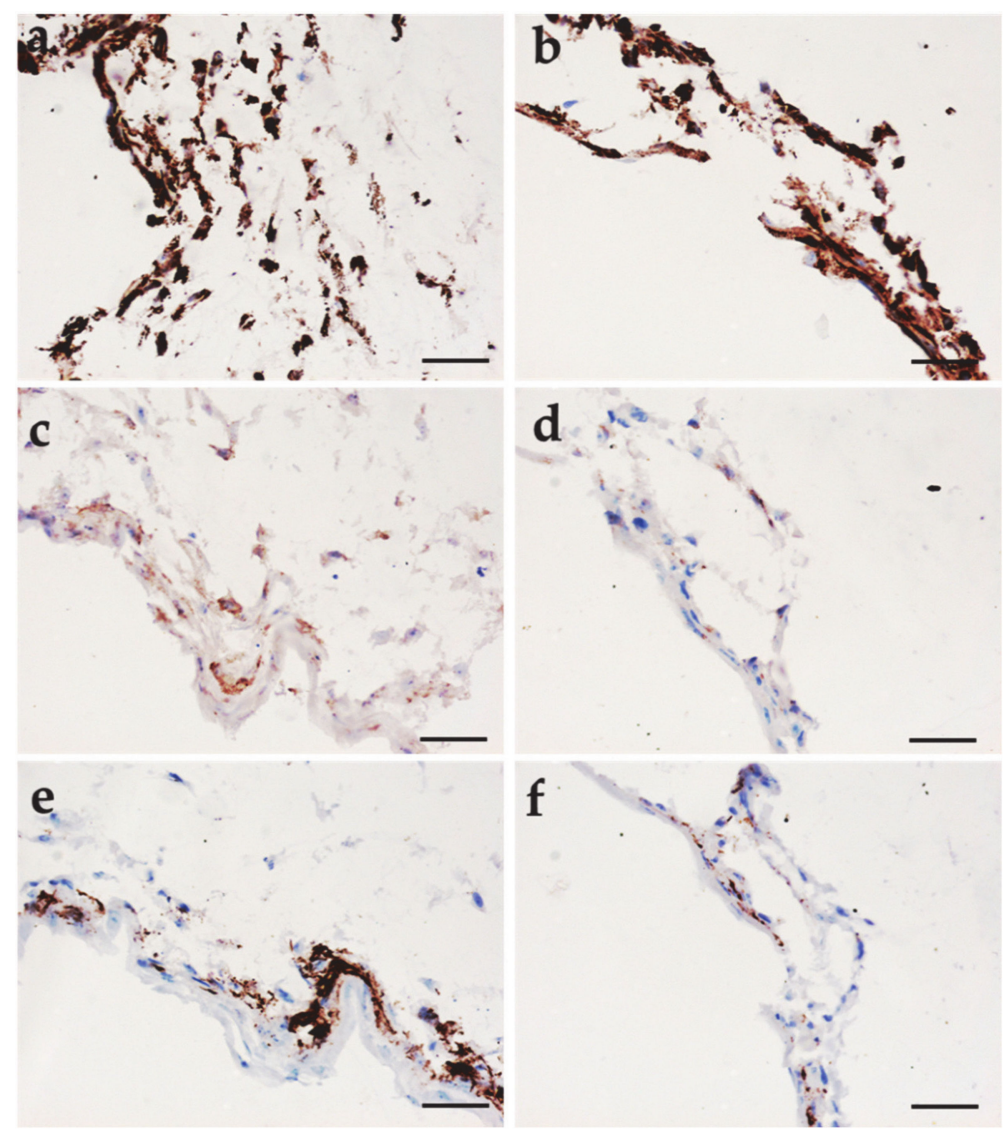

Figure 6. Immunohistochemical analysis of hMSC/*PCL constructs revealing the expression of collagen types I $(\mathbf{a}, \mathbf{b})$, III $(\mathbf{c}, \mathbf{d})$, and IV $(\mathbf{e}, \mathbf{f})$. Immunopositivity is in brown and cell nuclei are in violet: $(\mathbf{a}, \mathbf{c}, \mathbf{e})$ constructs differentiated in static conditions; $(\mathbf{b}, \mathbf{d}, \mathbf{f})$ construct differentiated in dynamic conditions. Scale bar is $50 \mu \mathrm{m}$; original magnification $400 \times$.

Very intense and comparable collagen type I expression was observed in the constructs differentiated both in static and dynamic conditions (Figure 6a,d). Collagen type III was instead weakly 
expressed, similarly by both construct types (Figure 6a,d). Collagen type IV was well expressed in both the construct types, being higher in statically than in dynamically differentiated samples. Interestingly, in both samples, Collagen type IV appeared located as in a basal lamina (Figure 6e,f).

We can summarize that hMSC/*PCL constructs cultured using a TM fibroblast-differentiation CM, developed as a variation of a chondrogenic-differentiation $\mathrm{CM}$, were able to promote the expression of the fibroblast surface maker, which resulted overexpressed. Collagen type II was also well expressed in these constructs as a synergistic effect of the differentiating $\mathrm{CM}$ and the bioreactor culture, which, in turn, was able to promote both these two TM-fibroblast key markers. The expression at protein level of other collagens-namely, type I and III-was not greatly different from those of the hMSC/*PCL constructs differentiated in static conditions, being collagen type I intensely expressed and the type III weakly expressed. Collagen type IV was expressed in both construct types resembling the localization found in the basal lamina of the basement membrane, but more intensely in statically cultured hMSC/*PCL constructs.

\subsection{Bioreactor Analysis}

This analysis served to evaluate the stress experienced by hMSCs during the dynamic culture, as a function of the scaffold physical and mechanical characteristics. Velocity streamlines during scaffold/holder system movement on the surface of the scaffold in contact with the fluid were evaluated during the culture time. When holder and scaffold were at start positions (at the inlet and at the end of the movement), a vortex occurred in the areas above and below the scaffold (Figure S2). Moreover, the maximum value of velocity appeared on the gap between the rings and tube walls. These results were in agreement with real situation. To analyze flow-dynamics on the scaffold, wall shear stress (WSS) was considered during the new-tissue growth, defined according to the following formula:

$$
W S S=\sqrt{\left(\tau_{x}\right)^{2}+\left(\tau_{y}\right)^{2}+\left(\tau_{z}\right)^{2}}
$$

being $\tau_{x}, \tau_{y}$ and $\tau_{z}$ the viscous stresses along the $x, y$ and $z$ directions, respectively. The WSS is the friction force generated by the ring motion on the surface of the scaffold in contact with the fluid, which, thus, affects cell growth. As the rings go up in their movement WSS increases (Figure S2a). Velocity field, as a result of fluid-structure simulation, is given in Figure S2c, showing similar vortex formation compared with velocity field resulting from fluid-dynamic simulation (Figure S2b), but also the higher value of velocity in the area below the scaffold. Mechanical stress increased along with the rising motion of the scaffold/holder system. As an example, the maximal von Mises stress in *PCL scaffold increases with time as follows: $(t=1.0 \mathrm{~s})>(\mathrm{t}=0.5 \mathrm{~s})>(\mathrm{t}=0 \mathrm{~s})$. HA/*PCL showed a similar trend, but the area sustaining the von Mises stress in the bottom position was wider than *PCL. A comparison of von Mises stresses for same positions showed that during its motion in the bioreactor, the *PCL scaffold is subject to a maximum stress that is double than that of HA/*PCL (Table 2). A von Mises stress map is shown in Figure 7.

Table 2. Von Mises stress (Pa) on the scaffold surfaces as calculated via Comsol multiphysics simulation.

\begin{tabular}{cccc}
\hline Time (s) & $\mathbf{0}$ & $\mathbf{0 . 5}$ & $\mathbf{1 . 0}$ \\
\hline *PCL & 0.0112 & 0.0626 & 0.0896 \\
HA $/{ }^{*} \mathrm{PCL}$ & 0.0070 & 0.0308 & 0.0411 \\
\hline
\end{tabular}


a

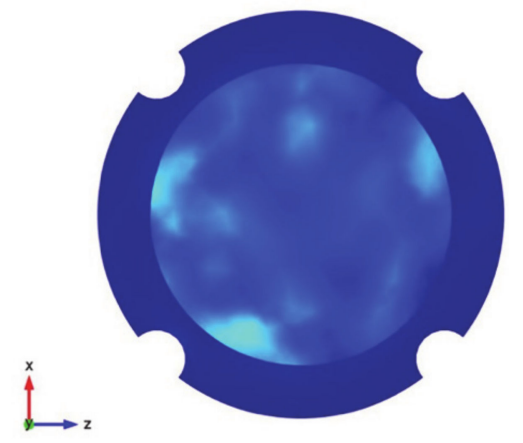

b

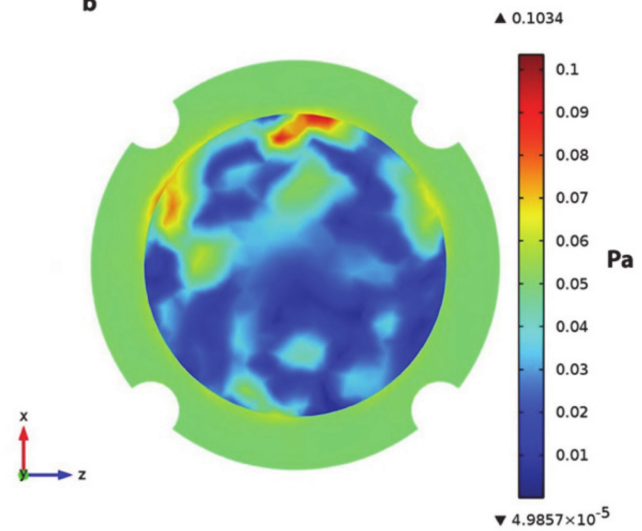

Figure 7. Comparison of von Mises stress map experienced by hMSCs on nanohydroxyapatite (HA) $/{ }^{*} \mathrm{PCL}(\mathbf{a})$ and ${ }^{*} \mathrm{PCL}(\mathbf{b})$ scaffolds in the TM bioreactor during the culture at $1.0 \mathrm{~s}$ from the start position $(\mathrm{t}=0 \mathrm{~s})$.

\subsection{Viability Assay and Immunohistochemical Analysis in the hMSC/HA/ ${ }^{*} P C L$ Constructs}

Representative results of hMSC/HA/*PCL mesh constructs are shown in Figure 8.
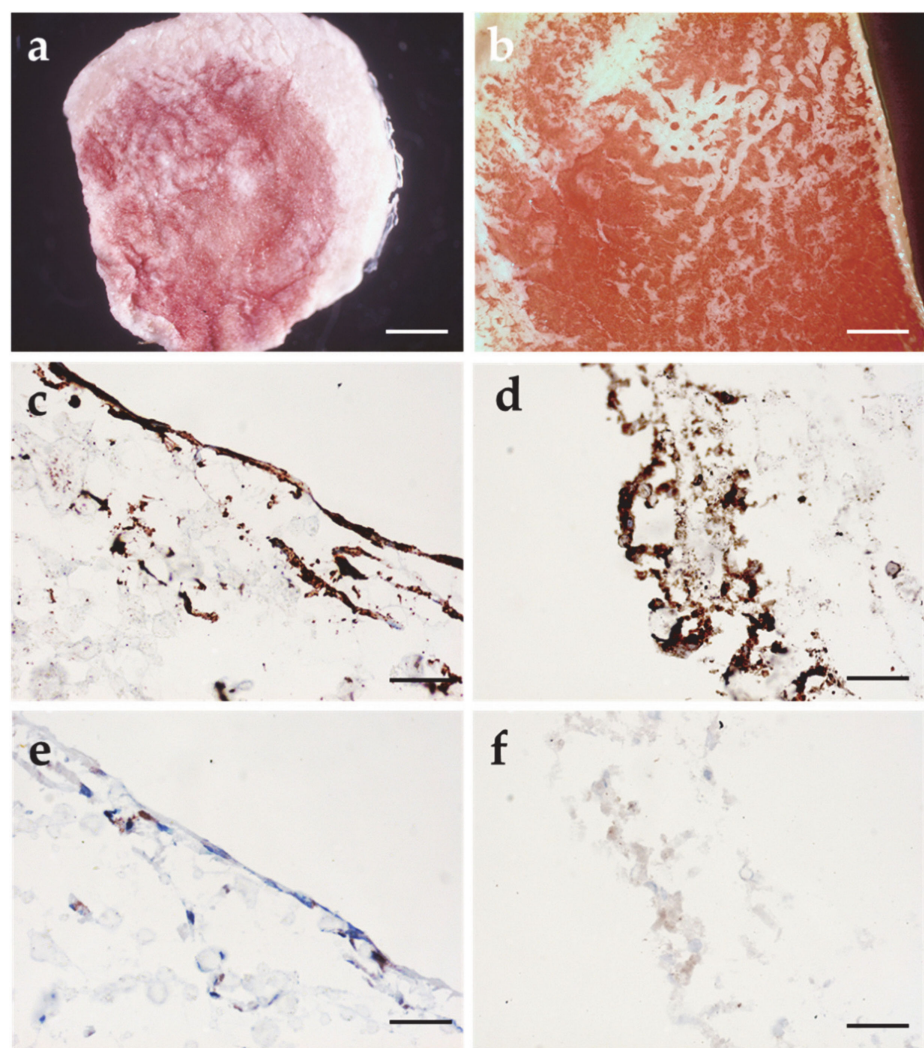

Figure 8. Neutral red assay and immunohistochemical analysis to detect fibroblast surface marker and collage type II on hMSC/HA/*PCL constructs: (a,c,e) statically differentiated; and, (b,d,f) dynamically differentiated. (a,b) Neutral red assay; viable cells are in red; $(\mathbf{c}-\mathbf{f})$ immunohistochemical analysis for: $(\mathbf{c}, \mathbf{d})$ fibroblast surface marker; and (e,f) collagen type II. Scale bars: (a,b) $1.2 \mathrm{~mm}$; (c-f) $50 \mu \mathrm{m}$ and original magnification $400 \times$.

This part was used to demonstrate that the mechanical properties and density of the scaffold can affect TM fibroblast differentiation of hMSCs as an effect of lower stress fields experienced by the cells in HA/ ${ }^{*}$ PCL scaffolds. Indeed, statistically significant differences in cell metabolic activity and cellularity were not detected by comparing hMSC/*PCL and hMSC/HA/ $/{ }^{*} \mathrm{PCL}$ constructs cultured 
in static conditions up to seven days of differentiation (Figure S3). Neutral red assay was used to compare cell viability and localization in static and dynamic cultures under a differentiative regimen and revealed good cell viability in all the tested constructs (Figure 8a,b). Immunohistochemistry highlighted, in both differentiated samples, a very intense positivity for the fibroblast surface marker (Figure $8 \mathrm{c}, \mathrm{d}$ ) and a very weak expression of collagen type II (Figure 8e,f). By using HA/*PCL scaffolds, no appreciable differences in term of antigenic expression were observed between static and dynamic culture conditions.

A semi-quantitative analysis of collagen immunopositivity obtained in the different hMSC/scaffold constructs is reported in Table 3. The most intense expression of collagen type II (scored as +++ ) was obtained in the native TM (Figure 5e).

Table 3. Semi-quantitative evaluation of the immunopositivity for TM collagens as expressed by different hMSC/scaffold ( ${ }^{*} \mathrm{PCL}$ or HA/*PCL) constructs after differentiation for seven days in different culture conditions (static or dynamic).

\begin{tabular}{ccccc}
\hline Collagens & ${ }^{*}$ PCL, Static & ${ }^{*}$ PCL, Dynamic & HA/*PCL, Static & HA/*PCL, Dynamic \\
\hline Type I & +++ & +++ & - & - \\
Type II & + & ++ & + & + \\
Type III & + & + & - & - \\
Type IV & $++/+++$ & ++ & - & - \\
\hline
\end{tabular}

\section{Discussion}

$\mathrm{TM}$ is the anatomic component of the ear, responsible for collecting the sound waves and transmitting them to the auditory bones. The repair and regeneration of the TM, as a consequence of traumas or infective pathologies, is still a subject of investigation. Until now, only a very limited number of studies have developed a straightforward tissue engineering approach for TM regeneration, but rather patches or stem cell injections have widely been promoted for in vivo applications, due to the fact that the TM is easily accessible and the damaged areas may be relatively small $[17,18]$. However, if the structural (i.e., collagenous) portion of the TM, i.e., the lamina propria of the pars tensa, has not properly healed, as occurs in large perforations, suboptimal hearing is observed [34]. At least two key factors have been identified to tightly link anatomy to physiology in the eardrum [1], and should be considered to design an effective tissue engineering strategy for TM regeneration: (1) the peculiar spatial arrangement of collagen fibers-radial and circular; and (2) the specific collagen types present in the native TM. Mastering both factors would allow a fully biomimetic and functional TM substitute to be generated in vitro, as the final outcome would be consistent with the natural tissue, from supramolecular down to molecular level. In this respect, the specific acousto-mechanical properties of collagen molecules could contribute to an optimal hearing process [35]. The first key factor has been investigated by recent studies, which demonstrated the feasibility of fabricating 3D printed eardrums with specific macroscale anatomic features, replicating some traits of radial and circular collagen pathways $[19,20]$. However, a route toward obtaining TM histological similarity in terms of collagen type expression by a cell source has not been reported so far. Our study aimed at modulating collagen type expression using the tissue engineering paradigm to address TM regeneration, thus facing the second key factor.

Immunohistochemical studies of the TM showed that, in the lamina propria of the pars tensa, the outer radial and inner circular fiber layers are formed of collagen types II and III to a larger extent with respect to the most common collagen type I [1]. The specific role of collagen type II in the eardrum is still unknown. Indeed, in other body domains collagen type II is not abundantly secreted by fibroblasts, but chondrocytes. For example, in tendons, collagen type II is found at the intersectoral zones with cartilage, whereas collagen type I accounts for $60-80 \%$ of collagens produced by specialized fibroblasts, called tenocytes [36]. In the cartilaginous tissue, the biomechanical role of collagen type II has been better described and is associated with the high resistance to deformation and fracture 
by virtue of collagen type II fiber interaction with proteoglycans [37]. Collagen type II fibers in the cartilaginous tissue have lower organization degrees than the ones usually found in type I fibers present in tendons and bone. Conversely, well organized collagen type II fibrils and fibers, as a result of fibroblast synthesis, have solely been found in the TM and in some other tissue structures of the inner ear, such as the tectorial membrane, the basilar membrane and other accessory structures $[12,38]$. Therefore, such a unique tissue characteristic provided by nature to the ear could be entitled with a peculiar acousto-mechanical function and replicating such a structure could provide an optimally engineered replacement. Unfortunately, human TM fibroblasts cannot easily be used for self-transplant procedures due to low availability and resulting tissue morbidity; as such, current in vitro studies mostly rely on culturing animal explants [39]. In contrast, dermal fibroblasts can be easily collected and rapidly overgrow in vitro, giving consistent multilayer structures in post-confluent conditions, by abundantly producing ECM components like collagen types I and III [40]. However, no evidence of controlling other collagen types has been reported for such cultures. In this scenario, stem cells, such as hMSCs, can offer a brilliant alternative, even though a specific differentiation pathway has to be set-up for TM fibroblast differentiation. This study specifically aimed at identifying biochemical, physical, and mechanical signals to induce the differentiation of hMSCs into fibroblasts synthesizing collagen type II, in addition to other collagens found in the eardrum, such as types I, III, and IV, which can spontaneously be secreted by other fibroblasts.

A TM-fibroblast differentiating CM was prepared with the purpose of promoting collagen type II expression, while maintaining the spindle-shaped morphotype proper of hMSCs, therefore their fibroblastic character. Specifically, a hand-prepared serum-free CM previously used for chondrogenic differentiation of hMSCs [41], was modified by the addition of FBS. It is recognized that serum-free 3D cultures enhance chondrogenic differentiation of hMSCs, with the production of collagen type II [42]. On the other hand, the lamina propria of the pars tensa is made up by fibroblasts, not chondrocytes. FBS was thus used to initiate cellular attachment to the substrate, facilitate cell proliferation and avoid a classic chondrogenic differentiation route by sustaining the fibroblastic morphotype [43]. In tissue culture plastics, hMSCs from the bone marrow express collagen type II gene, can synthetize the protein (mainly, tropocollagen II) in low amount, and do express the fibroblast surface antigen, as we also showed in this study (Figures $4 \mathrm{~d}$ and $5 \mathrm{~d}$ ). To test the efficacy of the differentiating cocktail, the expression of genes associated to the collagens of interest was evaluated by RT-PCR. The results showed that the application of different static and dynamic culture conditions and the administration of the differentiating CM influenced the collagen gene expression. Collage type I gene was less expressed in the samples cultivated in a static way, while collagen type II gene showed high expression in presence of differentiating $\mathrm{CM}$ without being significantly affected by culture condition mode. The expression of collagen type IV- $\alpha 1$ gene was higher in statically differentiated constructs, whereas type IV- $\alpha 2$ gene showed no statistically significant differences in all evaluated conditions.

The interaction with the scaffold is crucial for the cells to synthesize the ECM molecules, since it provides three-dimensional cues for their growth and differentiation. In this study, randomly oriented electrospun fibers based on *PCL were produced to obtain cell/scaffold constructs suitable for TM regeneration. In fact, the electrospinning process allows the fabrication of thin nonwovens made of ultrafine fibers that resemble the architecture of the fibrous ECM components of connective tissues, thus providing suitable physical stimuli for this specific application [27]. *PCL fiber meshes showed a Young's modulus of $3 \mathrm{MPa}$, whereas in normal TMs, it is found to be higher than $30 \mathrm{MPa}$, going up to $300 \mathrm{MPa}$, depending on the radial and circular direction and applied strain rates [44,45]. It is important to point out that the fully developed TM replacement will be mechanically reinforced by the cell-produced collagens. Therefore, we considered this value an acceptable starting point.

HMSCs were cultured on *PCL fiber meshes in static and dynamic environments, including undifferentiated and differentiated modes. The cells were viable, colonized uniformly the scaffold surface in any conditions, as confirmed by the Neutral Red assay, and adhered to the scaffold surface showing a remarkable expression of $f$-actin by the constructs under differentiation. This finding 
supported the role of the selected CM as a whole, and not only of FBS, which is present in both growth and differentiating $\mathrm{CM}$, towards the development of a mature fibroblast morphotype. The different samples showed a diverse morphology of $f$-actin filaments, appearing as parallel-stretched in statically differentiated, bundle-like in dynamically differentiated and disordered in the undifferentiated dynamic constructs. The difference in $f$-actin cytoskeleton between the statically and dynamically cultured samples was attributed to the dynamic forces present in the bioreactor. Regulation of cytoskeletal proteins, such as $f$-actin, enables physical cell processes, including cell shape and differentiation [46], which are greatly involved in the fibroblast-chondrocyte transformation [44]. The expression at a protein level of both differentiation markers, fibroblastic surface antigen and collagen type II, evaluated via immunohistochemistry, was also affected by the different culture conditions. The intensity of the fibroblastic surface antigen increased due to the differentiation cocktail and was further enhanced by the application of mechanical stimuli. Moreover, higher expression of collagen type II, with respect to other culture conditions, was only observed in the hMSC/*PCL constructs that underwent bioreactor culture, which ultimately resembled the expression found in human TM specimen. At a protein level, the expression of collagen types I and III was not modulated by the dynamic culture, resulting as intense as in static culture condition. Interestingly, in the differentiated constructs, collagen type IV localization resembled the one of the basal lamina, but it was more intensely expressed in statically cultured hMSC/*PCL constructs. This finding is in line with collagen type IV- $\alpha 1$ expression detected at a gene level. Several mechanisms regulate the expression of collagen type VI in fibroblasts, with respect to collagen types I and III. Collagen type IV expression has been indeed correlated to cell shape changes involving collagen VI as a cell-binding protein [47].

Altogether, these outcomes highlighted that by the use of *PCL electrospun scaffold, a properly supplemented CM and a TM bioreactor, it is possible to modulate collagen type II expression by fibroblast-differentiated hMSCs, which still produced other collagen types, such as types I, III and IV.

In order to better understand the cell response to the mechanical stimuli applied by the bioreactor, we produced a similar scaffold still based on *PCL, having slightly different physico-mechanical characteristics. This scaffold was obtained by adding HA nanoparticles to the polymer solution to be electrospun, which resulted in a lower density and lower elastic modulus (1.39 $\mathrm{MPa})$, than those measured in the plain *PCL scaffold. Alteration of polymer solution parameters has a direct effect on fiber size, which has been found to result in a different porosity, thus affecting the nonwoven density $[48,49]$. The decreased Young's modulus is suggestive that, in our experimental setting, due to insufficient interfacial properties, HA acted as a defect for the *PCL fibers, and not as a reinforcement, as reported in other nano-HA/PCL studies [22,50], thus reducing the stiffness of the nanocomposite [51]. It has to be considered that, for the same molecular weight, a star-branched polymer, like *PCL, accounts for many shorter polymer chains than its linear counterpart $[23,24]$. As a consequence, the polymer interaction with nanosized fillers could be reduced under mechanical loading. HA is naturally found in human body, so it is cytocompatible. Although in a former study HA was used to promote hMSC chondrogenesis using a polylactide electrospun scaffolds [52], in our experiments the primary role of HA in the nanocomposite fiber scaffold was purely physico-mechanical. The biological results showed a good cell viability and high scaffold surface colonization also in HA/*PCL scaffolds, demonstrated by the intensity and localization of Neutral Red dye. No difference in metabolic activity of the cells and cellularity (i.e., cell number) was detected between hMSC/*PCL and hMSC/HA/*PCL samples after one week. In this latter case, the fibroblast surface antigen resulted well expressed. Differently, collagen type II was not as intensely expressed as in hMSC/*PCL constructs. An in silico tool was used to evaluate the von Mises stress occurring on the scaffold during its motion in the bioreactor, which is the stress experienced by the differentiating cells laying on the nonwoven surface. As a result of the different scaffold properties, in the plain *PCL scaffold, von Mises stress resulted about two-fold higher than that calculated in the HA/*PCL counterpart. We thus concluded that not only a dynamic culture designed for TM cell/scaffold culture is needed, but a sufficient mechanical stress is fundamental to induce collagen type II synthesis by fibroblast-differentiating hMSCs. Many authors have addressed 
the influence of dynamic culture on stem cell fate, which has resulted to depend on several geometrical factors of the bioreactor/scaffold system, as a consequence of flow modification within the interspaces, and thus on the cell position and specific exposure to mechanical forces [53]. The key aspect of our device is its capacity of generating two stresses within the TM substitutes: pressure-induced (larger at the center) and hydrodynamic shear stresses (larger at the periphery). Such controlled mechanical stimuli, together with scaffold architectural cues and chemical stimuli experienced by the hMSCs during their maturation have been set-up to guide the TM tissue development from molecular scale (i.e., desired collagen type expression), up to possible microscale (i.e., appropriate spatial orientation of fibrils and fibers) [54]. For our specific system, we modeled a topographic map of the stress, which also varied in time according to the oscillatory motion of the bioreactor holder. This map highlighted areas of higher stress that may have produced circumferential-like aggregations of cells, visible by Neutral Red staining of hMSC/*PCL constructs (Figure $1 \mathrm{c}, \mathrm{d}$ ) and not found in hMSC/HA/*PCL counterpart (Figure 8a,b). This evidence deserves more investigation, as it could also underpin a zonal production of collagens, which may ultimately help in understanding how circular fibers have developed in the native TMs.

We demonstrated that in our in vitro system, electrospun ${ }^{*} \mathrm{PCL}$ scaffolds provided suitable properties for TM regeneration, in line with other investigators who have highlighted interesting outcomes in vivo using PCL family members. In particular, electrospun PCL/silk fibroin/umbilical cord serum fiber meshes have been reported to enable an efficient in vivo regeneration of subacute TM perforations in guinea pig models [55]. Similarly to PCL, the long biodegradation time shown by *PCL in vivo is expected to allow a sufficient timeframe for TM regeneration [56]. Indeed, residual infections and inflammatory processes connected to eardrum perforations, which usually lead to an aggressive environment for biomaterials, could also be controlled by the application of nanoparticles incorporated into polymer fibers for drug release in the middle up to the inner ear compartments [57,58].

Replicating a really biomimetic and autologous substitute for the eardrum via a tissue engineering approach will offer deaf people a better performing solution, thus contributing to improving their quality of life.

\section{Conclusions}

In this study, we showed the synergistic effect of hMSCs, electrospun *PCL scaffold and specific stimuli provided by a TM bioreactor and a differentiative cocktail, for eardrum regeneration. *PCL fiber meshes were able to support the growth of hMSCs. The combination of a TM-differentiating CM, the physico-mechanical properties of the *PCL scaffold and the mechanical stimuli provided by the TM bioreactor induced the expression of some molecular markers of interest for eardrum regeneration. Specifically, by this approach we were able to improve the protein level expression of collagen type II by fibroblast-differentiated hMSCs, as a consequence of the mechanical stress occurring at the scaffold surface. Only by combining a proper cell source, scaffold type, and stimulating factors-which are the three pillars of the tissue engineering paradigm - we succeeded in obtaining fibroblast-like cells producing collagen type II (in addition to other collagens). It is expected that, by tuning the parameters reported in this study, an optimal eardrum regeneration strategy could be developed, which will be useful in personalized medicine, as well as in understanding the complex physiology of the eardrum.

\section{Patents}

Apparatus and process for the preparation of a biomimetic tissue prosthesis of the tympanic membrane (WO/2015/040554).

Supplementary Materials: The following are available online at http:/www.mdpi.com/2076-3417/10/9/3043/s1, Supplementary document including: Figure S1. Scaffold morphology.; Figure S2. Velocity field fluid-structure simulation.; Figure S3: hMSC metabolic activity and cellularity on *PCL-based scaffolds.; Tables S1 and S2: Physical and mechanical properties of the scaffolds. 
Author Contributions: Data curation D.D.; Formal analysis D.D., V.G. and M.M.; Funding acquisition S.D., S.B., and C.S.; Investigation S.M., A.R. and D.P.; Methodology F.C. and M.P.; Project administration S.D.; Resources M.P., S.B., C.S. and F.C.; Supervision S.M. and S.D.; Validation D.D.; Visualization D.D. and S.D.; Writing-Original draft D.D. and S.D.; Writing-Review and editing D.D., S.D., D.P., F.C., M.M. and V.G. All authors have read and agreed to the published version of the manuscript.

Funding: This research was funded by Tuscany Region (Italy), grant "Toscana Salute 2009" and partially by EuroNanoMed III 2017, project 4NanoEARDRM (for Italian partners, co-funded by Italian Ministry of University and Research; MIUR).

Acknowledgments: The authors wish to acknowledge Stefano Landi (Dept. of Biology, University of Pisa) for his support to RT-PCR, Luisa Trombi (Dept. of Clinical and Experimental Medicine, University of Pisa) for her technical support in hMSC isolation, and Dinuccio Dinucci (Dept. of Chemistry, University of Pisa) for his technical support to CLSM analysis.

Conflicts of Interest: The authors declare no conflict of interest.

\section{References}

1. Lim, D.J. Structure and function of the tympanic membrane: A review. Acta Oto Rhino Laryngol. Belg. 1995, 49, 101-115.

2. Milazzo, M.; Fallah, E.; Carapezza, M.; Kumar, N.S.; Lei, J.H.; Olson, E.S. The path of a click stimulus from ear canal to umbo. Hear. Res. 2017, 346, 1-13. [CrossRef] [PubMed]

3. Mallo, M. Embryological and genetic aspects of middle ear development. Int. J. Dev. Boil. 1998, 42, 11-22.

4. Lim, D.J. Tympanic membrane: Electron microscopic observation part I: Pars tensa. Acta Oto Laryngol. 1968, 66, 181-198. [CrossRef]

5. Lim, D.J. Human tympanic membrane: An ultrastructural observation. Acta Oto Laryngol. 1970, 70, $176-186$. [CrossRef]

6. Shimada, T.; Lim, D.J. The fiber arrangement of the human tympanic membrane. A scanning electron microscopic observation. Ann. Otol. Rhinol. Laryngol. 1971, 80, 210-217. [CrossRef]

7. Fay, J.P.; Puria, S.; Steele, C.R. The discordant eardrum. Proc. Natl. Acad. Sci. USA 2006, 103, $19743-19748$. [CrossRef] [PubMed]

8. Knutsson, J.; Bagger-Sjöbäck, D.; Von Unge, M. Collagen type distribution in the healthy human tympanic membrane. Otol. Neurotol. 2009, 30, 1225-1229. [CrossRef]

9. Stenfeldt, K.; Johansson, C.; Hellström, S. The collagen structure of the tympanic membrane: Collagen types I, II, and III in the healthy tympanic membrane, during healing of a perforation, and during infection. Arch. Otolaryngol. Head Neck Surg. 2006, 132, 293. [CrossRef]

10. Broekaert, D. The tympanic membrane: A biochemical updating of structural components. Acta Oto Rhino Laryngol. Belg. 1995, 49, 127-137.

11. Mallo, M. Formation of the middle ear: Recent progress on the developmental and molecular mechanisms. Dev. Boil. 2001, 231, 410-419. [CrossRef] [PubMed]

12. Slepecky, N.B.; Savage, J.E.; Yoo, T.J. Localization of type II, IX and V collagen in the inner ear. Acta Oto Laryngol. 1992, 112, 611-617. [CrossRef]

13. Hardman, J.; Muzaffar, J.; Nankivell, P.; Coulson, C. Tympanoplasty for chronic tympanic membrane perforation in children: Systematic review and meta-analysis. Otol. Neurotol. 2015, 36, 796-804. [CrossRef] [PubMed]

14. Lerut, B.; Pfammatter, A.; Moons, J.; Linder, T. Functional correlations of tympanic membrane perforation size. Otol. Neurotol. 2012, 33, 379-386. [CrossRef] [PubMed]

15. Jalali, M.M.; Motasaddi, M.; Kouhi, A.; Dabiri, S.; Soleimani, R. Comparison of cartilage with temporalis fascia tympanoplasty: A meta-analysis of comparative studies. Laryngoscope 2016, 127, 2139-2148. [CrossRef]

16. Lee, P.; Kelly, G.; Mills, R.P. Myringoplasty: Does the size of the perforation matter? Clin. Otolaryngol. Allied Sci. 2002, 27, 331-334. [CrossRef]

17. Teh, B.M.; Marano, R.J.; Shen, Y.; Friedland, P.L.; Dilley, R.J.; Atlas, M.D. Tissue Engineering of the Tympanic Membrane. Tissue Eng. Part B Rev. 2013, 19, 116-132. [CrossRef]

18. Villar-Fernandez, M.A.; Lopez-Escamez, J.A. Outlook for tissue engineering of the tympanic membrane. Audiol. Res. 2015, 5. [CrossRef] 
19. Mota, C.; Danti, S.; D’Alessandro, D.; Trombi, L.; Ricci, C.; Puppi, D.; Dinucci, D.; Milazzo, M.; Stefanini, C.; Chiellini, F.; et al. Multiscale fabrication of biomimetic scaffolds for tympanic membrane tissue engineering. Biofabrication 2015, 7, 025005. [CrossRef]

20. Kozin, E.D.; Black, N.L.; Cheng, J.T.; Cotler, M.J.; McKenna, M.J.; Lee, D.J.; Lewis, J.A.; Rosowski, J.J.; Remenschneider, A.K. Design, fabrication, and in vitro testing of novel three-dimensionally printed tympanic membrane grafts. Hear. Res. 2016, 340, 191-203. [CrossRef]

21. Danti, S.; D'Alessandro, D.; Mota, C.; Bruschini, L.; Berrettini, S. Applications of bioresorbable polymers in skin and eardrum. In Bioresorbable Polymers for Biomedical Applications: From Fundamentals to Translational Medicine; Elsevier: Amsterdam, The Netherlands, 2016; ISBN 9780081002667.

22. Milazzo, M.; Contessi Negrini, N.; Scialla, S.; Marelli, B.; Farè, S.; Danti, S.; Buehler, M.J. Additive Manufacturing Approaches for Hydroxyapatite-Reinforced Composites. Adv. Funct. Mater. 2019, 29. [CrossRef]

23. Burchard, W. Solution Properties of Branched Macromolecules. In Branched Polymers II; Springer: Berlin/Heidelberg, Germany, 1999; ISBN 978-3-540-65005-8.

24. Puppi, D.; Detta, N.; Piras, A.M.; Chiellini, F.; Clarke, D.A.; Reilly, G.C.; Chiellini, E. Development of electrospun three-arm star poly( $\varepsilon$-caprolactone) meshes for tissue engineering applications. Macromol. Biosci. 2010, 10, 887-897. [CrossRef]

25. Balakrishnan, P.; Gardella, L.; Forouharshad, M.; Pellegrino, T.; Monticelli, O. Star poly(e-caprolactone)-based electrospun fibers as biocompatible scaffold for doxorubicin with prolonged drug release activity. Colloids Surf. B Biointerfaces 2018, 161, 488-496. [CrossRef] [PubMed]

26. Buehler, M.J. Nature designs tough collagen: Explaining the nanostructure of collagen fibrils. Proc. Natl. Acad. Sci. USA 2006, 103, 12285-12290. [CrossRef] [PubMed]

27. Danti, S.; Mota, C.; D’alessandro, D.; Trombi, L.; Ricci, C.; Redmond, S.L.; De Vito, A.; Pini, R.; Dilley, R.J.; Moroni, L.; et al. Tissue engineering of the tympanic membrane using electrospun PEOT/PBT copolymer scaffolds: A morphological in vitro study. Hear. Balance Commun. 2015, 13, 133-147. [CrossRef]

28. Lee, C.H.; Moioli, E.K.; Mao, J.J. Fibroblastic differentiation of human mesenchymal stem cells using connective tissue growth factor. In Proceedings of the Annual International Conference of the IEEE Engineering in Medicine and Biology, New York, NY, USA, 30 August-3 September 2006.

29. Tew, S.R.; Murdoch, A.D.; Rauchenberg, R.P.; Hardingham, T.E. Cellular methods in cartilage research: Primary human chondrocytes in culture and chondrogenesis in human bone marrow stem cells. Methods 2008, 45, 2-9. [CrossRef] [PubMed]

30. Gaspar, D.A.; Gomide, V.; Monteiro, F.J. The role of perfusion bioreactors in bone tissue engineering. Biomatter 2013, 2, 167-175. [CrossRef]

31. Puppi, D.; Piras, A.M.; Detta, N.; Dinucci, D.; Chiellini, F. Poly (lactic-co-glycolic acid) electrospun fibrous meshes for the controlled release of retinoic acid. Acta Biomater. 2010, 6, 1258-1268. [CrossRef]

32. Trombi, L.; Danti, S.; Savelli, S.; Moscato, S.; D'Alessandro, D.; Ricci, C.; Giannotti, S.; Petrini, M. Mesenchymal Stromal Cell Culture and Delivery in Autologous Conditions: A Smart Approach for Orthopedic Applications. J. Vis. Exp. 2016, e54845. [CrossRef]

33. Pfaffl, M.W. A new mathematical model for relative quantification in real-time RT-PCR. Nucleic Acids Res. 2001, 29, e45. [CrossRef]

34. Lou, Z.-C.; Lou, Z.-H.; Zhang, Q.-P. Traumatic tympanic membrane perforations: A study of etiology and factors affecting outcome. Am. J. Otolaryngol. 2012, 33, 549-555. [CrossRef]

35. Milazzo, M.; Jung, G.S.; Danti, S.; Buehler, M.J. Wave propagation and energy dissipation in collagen molecules. ACS Biomater. Sci. Eng. 2020, 6, 1367-1374. [CrossRef]

36. Fukuta, S.; Oyama, M.; Kavalkovich, K.; Fu, F.H.; Niyibizi, C. Identification of types II, IX and X collagens at the insertion site of the bovine achilles tendon. Matrix Biol. 1998, 17, 65-73. [CrossRef]

37. Andriamanalijaona, R. Cell therapies for articular cartilage repair: Chondrocytes and mesenchymal stem cells. In Regenerative Medicine and Biomaterials for the Repair of Connective Tissues; Elsevier: Amsterdam, The Netherlands, 2010; pp. 266-300.

38. Thalmann, I. Collagen of accessory structures of organ of Corti. Connect. Tissue Res. 1993, 29, $191-201$. [CrossRef] [PubMed]

39. Liew, L.J.; Day, R.M.; Dilley, R.J. Tympanic membrane organ culture using cell culture well inserts engrafted with tympanic membrane tissue explants. BioTechniques 2017, 62, 109-114. [CrossRef] 
40. Dumas, M.; Chaudagne, C.; Bonté, F.; Meybeck, A. In vitro biosynthesis of type I and III collagens by human dermal fibroblasts from donors of increasing age. Mech. Ageing Dev. 1994, 73, 179-187. [CrossRef]

41. Danti, S.; D'Acunto, M.; Trombi, L.; Berrettini, S.; Pietrabissa, A. A Micro/Nanoscale Surface Mechanical Study on Morpho-Functional Changes in Multilineage-Differentiated Human Mesenchymal Stem Cells. Macromol. Biosci. 2007, 7, 589-598. [CrossRef]

42. Ho, S.T.B.; Tanavde, V.M.; Hui, J.H.; Lee, E.H. Upregulation of adipogenesis and chondrogenesis in MSC serum-free culture. Cell Med. 2011, 2, 27-42. [CrossRef]

43. Von Der Mark, K.; Gauss, V.; Von Der Mark, H.; Müller, P. Relationship between cell shape and type of collagen synthesised as chondrocytes lose their cartilage phenotype in culture. Nature 1977, 267, 531-532. [CrossRef]

44. Fay, J.; Puria, S.; Decraemer, W.F.; Steele, C. Three approaches for estimating the elastic modulus of the tympanic membrane. J. Biomech. 2005, 38, 1807-1815. [CrossRef]

45. Luo, H.; Dai, C.; Gan, R.Z.; Lu, H. Measurement of Young's modulus of human tympanic membrane at high strain rates. J. Biomech. Eng. 2009, 131, 064501. [CrossRef] [PubMed]

46. Stricker, J.; Falzone, T.; Gardel, M.L. Mechanics of the F-actin cytoskeleton. J. Biomech. 2010, 43, 9-14. [CrossRef] [PubMed]

47. Hatamochi, A.; Aumailley, M.; Mauch, C.; Chu, M.L.; Timpl, R.; Krieg, T. Regulation of collagen VI expression in fibroblasts. Effects of cell density, cell-matrix interactions, and chemical transformation. J. Biol. Chem. 1989, 264, 3494-3499. [PubMed]

48. Haider, A.; Haider, S.; Kang, I.-K. A comprehensive review summarizing the effect of electrospinning parameters and potential applications of nanofibers in biomedical and biotechnology. Arab. J. Chem. 2018, 11, 1165-1188. [CrossRef]

49. Pham, Q.P.; Sharma, U.; Mikos, A.G. Electrospun poly ( $\varepsilon$-caprolactone) microfiber and multilayer nanofiber/microfiber scaffolds: Characterization of scaffolds and measurement of cellular infiltration. Biomacromolecules 2006, 7, 2796-2805. [CrossRef] [PubMed]

50. Mochane, M.J.; Motsoeneng, T.S.; Sadiku, E.R.; Mokhena, T.C.; Sefadi, J.S. Morphology and Properties of Electrospun PCL and Its Composites for Medical Applications: A Mini Review. Appl. Sci. 2019, 9, 2205. [CrossRef]

51. Levita, G.; Marchetti, A.; Lazzeri, A. Fracture of ultrafine calcium carbonate/polypropylene composites. Polym. Compos. 1989, 10, 39-43. [CrossRef]

52. Spadaccio, C.; Rainer, A.; Trombetta, M.; Vadalá, G.; Chello, M.; Covino, E.; Denaro, V.; Toyoda, Y.; Genovese, J.A. Poly-L-lactic acid/hydroxyapatite electrospun nanocomposites induce chondrogenic differentiation of human MSC. Ann. Biomed. Eng. 2009, 37, 1376-1389. [CrossRef]

53. Yeatts, A.B.; Geibel, E.M.; Fears, F.F.; Fisher, J.P. Human mesenchymal stem cell position within scaffolds influences cell fate during dynamic culture. Biotechnol. Bioeng. 2012, 109, 2381-2391. [CrossRef]

54. Limongi, T.; Tirinato, L.; Pagliari, F.; Giugni, A.; Allione, M.; Perozziello, G.; Candeloro, P.; Di Fabrizio, E. Fabrication and applications of micro/nanostructured devices for tissue engineering. Nano Micro Lett. 2017, 9, 1. [CrossRef]

55. Lee, H.; Jang, C.H.; Kim, G.H. A polycaprolactone/silk-fibroin nanofibrous composite combined with human umbilical cord serum for subacute tympanic membrane perforation; an in vitro and in vivo study. J. Mater. Chem. B 2014, 2, 2703-2713. [CrossRef] [PubMed]

56. Dini, F.; Barsotti, G.; Puppi, D.; Coli, A.; Briganti, A.; Giannessi, E.; Miragliotta, V.; Mota, C.; Pirosa, A.; Stornelli, M.R.; et al. Tailored star poly ( $\varepsilon$-caprolactone) wet-spun scaffolds for in vivo regeneration of long bone critical size defects. J. Bioact. Compat. Polym. 2016, 31, 15-30. [CrossRef]

57. Günday, C.; Anand, S.; Gencer, H.B.; Munafò, S.; Moroni, L.; Fusco, A.; Donnarumma, G.; Ricci, C.; Hatir, P.C.; Türeli, N.G.; et al. Ciprofloxacin-loaded polymeric nanoparticles incorporated electrospun fibers for drug delivery in tissue engineering applications. Drug Deliv. Transl. Res. 2020, 1-15. [CrossRef] [PubMed]

58. Valente, F.; Astolfi, L.; Simoni, E.; Danti, S.; Franceschini, V.; Chicca, M.; Martini, A. Nanoparticle drug delivery systems for inner ear therapy: An overview. J. Drug Deliv. Sci. Technol. 2017, 39, 28-35. [CrossRef]

(C) 2020 by the authors. Licensee MDPI, Basel, Switzerland. This article is an open access article distributed under the terms and conditions of the Creative Commons Attribution (CC BY) license (http://creativecommons.org/licenses/by/4.0/). 